\title{
Phosphorus-31 Solid-State NMR Study of Iminophosphines: Influence of Electronic Structure and Configuration of the Double Bond on Phosphorus Shielding
}

\author{
D. Gudat, ${ }^{\circ \dagger}$ W. Hoffbauer, ${ }^{\dagger}$ E. Niecke, ${ }^{\circ \dagger}$ W. W. Schoeller, ${ }^{\ddagger}$ U. Fleischer, ${ }^{\natural}$ and \\ W. Kutzelnigg?
}

Contribution from the Institut für Anorganische Chemie der Universität Bonn, Gerhard Domagk Strasse 1, D-53121 Bonn, FRG, Fakultât für Chemie der Universität Bielefeld, Postfach 100131, D-33501 Bielefeld, FRG, and Lehrstuhl für Theoretische Chemie, Ruhr-Universität Bochum, D-44780 Bochum, FRG

Received March 23, $1994^{\circ}$

\begin{abstract}
The principal elements of the ${ }^{31} \mathrm{P}$ nuclear magnetic shielding tensors of iminophosphines of the type $\mathrm{R}-\mathrm{P}=\mathrm{N}-\operatorname{aryl}\left(\operatorname{aryl}=\mathrm{C}_{6} \mathrm{H}_{2} \mathrm{tBu} ; \mathrm{R}=\mathrm{tBu}, \mathrm{Ph}_{3} \mathrm{Sn}(\mathrm{tBu}) \mathrm{N}\right.$, aryl- $\mathrm{N}=\mathrm{P}-(\mathrm{tBu}) \mathrm{N}$, aryl $-\mathrm{NH}$, aryl- $\mathrm{N}=\mathrm{P}-$ (adamantyl)N, $\mathrm{Ph}_{2} \mathrm{~N}$, carbazolyl, $\mathrm{tBu}_{2} \mathrm{C}=\mathrm{N}$, iminofluorenyl, $\mathrm{Cl}, \mathrm{Br}, \mathrm{I} ; \mathrm{5}-16$ ) are determined from high-resolution solid-state MAS (magic angle spinning) NMR spectra. Comparison of the data with the results of IGLO calculations on shielding tensors of model compounds is used to assign the orientation of the principal axes system in the molecular frame. The observed shieldings can be correlated with structural features of the compounds. In $(E)$-configured alkyland amino-iminophosphines, the component at high field $\left(\delta_{33}\right)$ is approximately perpendicular to the RPN plane and the low-field component $\left(\delta_{11}\right)$ is in this plane and close to the $\mathrm{P}-\mathbf{R}$ bond. Introduction of $\pi$-donor substituents $\left(\mathbf{R}_{2}^{\prime} \mathrm{N}\right)$ at phosphorus produces large increases in $\delta_{11}$, which can be related to corresponding changes in the $n-\pi^{*}$ electronic transition energies. A similar effect is found for a change of the double-bond geometry from $(E)$-to $(Z)$-configuration. Electronegative halogen substituents $(\mathrm{Cl}, \mathrm{Br})$ give rise to a further substantial increase in the tensor component at high field, $\delta_{33}$. Furthermore, a change of the orientation of the principal axes system in the molecular frame occurs, with $\delta_{33}$ being aligned close to the P,N bond axis and the intermediate component, $\delta_{22}$, perpendicular to the RPN plane. A discussion of these effects is given in terms of partial triple-bond character for the $\mathrm{P}, \mathrm{N}$ bond. The shape of the shielding tensor of the iodo compound (16) suggests that the electronic environment of the $P, N$ moiety is similar to other systems containing a genuine triple bond. The unique difference between the isotropic chemical shifts in the solid state and in solution $(\Delta \delta 118 \mathrm{ppm})$ in this case indicates that this phenomenon is confined to the solid state and can be attributed to the substantial polarization of the P,I bond, which is a consequence of the observed intermolecular association.
\end{abstract}

\section{Introduction}

The development of a chemistry of multiple bond systems, involving heavier main group elements can be considered as one of the most exciting advances in main group chemistry during the last two decades. Among the many stable multiple bond systems which have been successfully characterized, compounds with ( $p$ p) $\pi$-bonds to phosphorus were among the first stable examples to be synthetized ${ }^{1}$ and still play an important role in this field. ${ }^{2}$ The early success in this area may to a great part be attributed to the easy NMR observability of ${ }^{31} \mathrm{P}$, providing synthetic chemists with a sensitive analytical probe. In contrast to the routine nature of solution ${ }^{31}$ P-NMR, however, rather few solid-state NMR investigations have yet been carried out, and the first studies focused mainly on multiple bonding between phosphorus with another phosphorus (aryl- $\mathrm{P}=\mathrm{P}-\operatorname{aryl} 1^{3,4}(1)$; aryl $=2,4,6$-tristert-butylphenyl)) or carbon (aryl- $\mathrm{P}=\mathrm{C}\left(\mathrm{SiMe}_{3}\right)_{2}(2)$, aryl$\left.\mathrm{C} \equiv \mathrm{P}(3)^{5}\right)$. The results of these studies nevertheless yielded valuable information on the nature of the multiple bonding which

$\uparrow$ Universităt Bonn.

Universităt Bielefeld.

I Ruhr-Universitất Bochum.

- Abstract published in Advance ACS Abstracts, July 1, 1994

(1) (a) Gier, T. E. J. Am. Chem. Soc. 1961, 83, 1769. (b) Dimroth, K.; Hoffmann, P. Angew. Chem., Int. Ed. Engl. 1964, 3, 384.

(2) For a review, see: Multiple Bonds and Low Coordination in Phosphorus Chemistry; Regitz, M., Scherer, O., Eds.; Georg Thieme Verlag: Stuttgart, 1990.

(3) Zilm, K. W.; Webb, G. G.; Cowley, A. H.; Pakulski, M.; Orendt, A. J. Am. Chem. Soc. 1988, 110, 2032.

(4) Challoner, R.; McDowell, C. A. J. Chem. Phys. 1991, 94, 7038.

(5) Duchamp, J. C.; Pakulski, M.; Cowley, A. H.; Zilm, K. W. J. Am. Chem. Soc. 1990, 112, 6803 complements material available from other sources (mostly X-ray diffraction methods).

Iminophosphines featuring ( $3 p-2 p) \pi$-bonding between phosphorus and nitrogen occupy a special place among the numerous multiple bond systems because of the potential of the iminonitrogen to vary its bonding state by means of formal electron release or acceptance., ${ }^{2,6}$ This results in a wide range of different structures of the multiple bond system, which in turn can be held responsible for the observed very intricate and diverse chemical reactivity. ${ }^{2,6} \mathrm{~A}$ particularly interesting aspect is the phenomenon of $(E / Z)$-double-bond isomerism, since it has been shown both by experiment ${ }^{2,6}$ and theory ${ }^{7}$ that inversion of the double-bond configuration is followed by considerable changes in the bonding parameters. Regarding the large chemical shift anisotropies of phosphorus nuclei in multiple bond systems ${ }^{3-5}$ as well as the sensitivity of the individual components of the shielding tensor toward changes in electron distribution and molecular geometry, solid-state NMR spectroscopy seems a promising tool to gain further understanding of the bonding situation in iminophosphines. Its potential is clearly emphasized in the recent investigation of the solid-state NMR parameters of the cation aryl- N $\equiv \mathrm{P}^{+}(4),{ }^{8}$ which is currently the only phosphorus nitrogen multiple bond system having been studied with this method.

We have set out to study the phosphorus chemical shielding in a series of differently substituted iminophosphanes with both

(6) Niecke, E.; Gudat, D. Angew. Chem., Int. Ed. Engl. 1991, 30, 217 (7) (a) Trinquier, G. J. Am. Chem. Soc. 1982, 104, 6969. (b) Nguyen, M. T.; McGinn, M. A.; Hegarty, A. F. J. Am. Chem. Soc. 1985, 107, 8029. (8) Curtis, R. D.; Schriver, M. J.; Wasylishen, R. E. J. Am. Chem. Soc $1991,113,1493$ 
$(E)$ - and $(Z)$-configuration of the double bond by high-resolution magic angle spinning (MAS) solid-state NMR spectroscopy. Comparison of the magnitudes of the principal components of the shielding tensor as obtained from the experiments with calculated (IGLO ${ }^{9}$ ) data of model compounds yields information about the orientation of the shielding tensor in the molecular frame. The implications of variations in the electronic structure of the double bond on the chemical shielding will be discussed on the basis of both experimental and theoretical data and will be related to the NMR properties of other known phosphoruscontaining multiple bond systems.

Methodology. For complete characterization of the magnetic shielding tensor of a nucleus in a molecule, knowledge of both the magnitudes and orientations of the three principal components has to be gained. Whereas the magnitudes are easily obtained from the solid-state NMR spectra of powdered samples, ${ }^{10,11}$ determination of the tensor orientation generally requires the investigation of single crystals; ${ }^{11}$ in favorite cases, when isolated AX spin pairs with dipolar coupling are present, these data are partially accessible from polycrystalline samples by dipolar chemical shift spectroscopy. 12

In the case of the studied iminophosphanes, the presence of additional quadrupolar nuclei gives rise to a significant complication in the solid-state ${ }^{31} \mathrm{P}-\mathrm{NMR}$ spectra since the observed line shapes depend on scalar and dipolar couplings as well as quadrupolar interactions, in addition to the effect of chemical shielding. Except in highly symmetric molecules, the number of observable discontinuities in wide-line spectra of solid samples is normally insufficient to afford complete analysis of all interactions. In order to facilitate a straightforward extraction of shielding tensor data, it seems therefore advantageous to reduce or eliminate the additional perturbations. For instance, quadrupolar interactions with abundant ${ }^{14} \mathrm{~N}$ nuclei may be removed by isotopic labeling with ${ }^{15} \mathrm{~N}(I=1 / 2)$; however, the use of this method is limited because suitable ${ }^{15} \mathrm{~N}$-doped precursors may be unreasonably expensive or not available at all.

Using a different approach, averaging of anisotropic dipolar interactions in solid-state NMR spectra of spin- $1 / 2$ nuclei can be afforded by $\mathrm{MAS}^{13}$ at adequate speed. The accessible rotation rates $(1-20 \mathrm{kHz})$ generally suffice to eliminate effects of dipolar interactions unless strong coupling is present, ${ }^{14}$ but are for many inorganic compounds still in the slow-spinning regime when compared to the shielding tensor. The magnitudes of the principal tensor components may then be derived by intensity analysis of the observed spinning sideband patterns. ${ }^{15,16}$ Interactions with adjacent quadrupolar nuclei are scaled down by MAS, but their effects cannot be completely eliminated because their angular dependence is not averaged to $0.17,18$ As a consequence, MAS spectra show residual dipolar coupling effects in the form of characteristic fine structure of the resonance lines. Theoretical analyses of these effects have been carried out but are rather complicated $; 17$ when the quadrupolar interactions are weak as compared to the Zeeman energies (this is generally found for spin- $1 / 2$ nuclei coupling to ${ }^{14} \mathrm{~N}$ ), the treatment can be considerably simplified by a first-order perturbation approach. ${ }^{18}$ For a concise

(9) (a) Kutzelnigg, W. Isr. J. Chem. 1980, 19, 193. (b) Schindler, M. S.; Kutzelnig8, W.J. Chem. Phys. 1982, 76, 1919. (c) Kutzelnigg, W.; Fleischer, U.; Schindler, M. In NMR Basic Principles and Progress, Diehl, P., Fluck, E., Kosfeld, R., Eds.; Springer Verlag: Berlin, 1990; Vol. 23, p $167 \mathrm{ff}$.

(10) Taylor, P. C.; Baugher, J. F.; Kriz, H. M. Chem. Rev. 1975, 75, 203.

(11) Veeman, W. S. Prog. Nucl. Magn. Reson. Spectrosc. 1984, 16, 193.

(12) Power, W. P.; Wasylishen, R. E. In Annual Reports on NMR Spectroscopy; Webb, G. A., Ed.; Academic Press: London, 1991; p 1 ff. (13) (a) Andrew, E. R.; Bradbury, A.; Eades, R. G. Nature 1958, 182 1659. (b) Ibid. 1959, 183, 1802. (c) Lowe, I. J. Phys. Rev. Lett. 1959, 2 , 285 .

(14) Wu, G.; Wasylishen, R. E.; Curtis, R. D. Can. J. Chem. 1992, 70, 863

(15) Maricq, M. M.; Waugh, J. S. J. Chem. Phys. 1979, 70, 3300

(16) Herzfeld, J.; Berger, A. E. J. Chem. Phys. 1980, 73, 6021.

(17) Zumbulyadis, N.; Henrichs, P. M.; Young, R. H. J. Chem. Phys. $1981,75,1603$. 50.

(18) Ólivieri, A. C.; Frydman, L.; Diaz, L. E. J. Magn. Reson. 1987, 75, determination of the shielding tensor from MAS spectra, the residual dipolar interactions must be taken into account. Gobetto et al. ${ }^{19}$ have shown that for IS spin systems with axial symmetry (the shielding tensors $\sigma(I), \sigma(S)$ are coaxial with both the dipolar and scalar coupling tensors $\mathbf{D}_{1 \mathrm{~S}}$ and $\mathbf{J}_{\mathrm{IS}}$, and all tensors have axial symmetry) each spin state $\mathrm{m}_{i}$ of the quadrupolar spin $S$ gives rise to a separate spinning sideband manifold in the spectrum of $I$. These subspectra can be described by effective shielding tensors $\sigma^{\prime}(I)$ with anisotropies $\sigma_{i}^{\prime}(I)=\sigma(I)-2 D_{\mathrm{IS}}^{\prime} \mathrm{m}_{i}(S) / \nu\left(D_{\mathrm{IS}}^{\prime}=\right.$ effective dipolar coupling constant, $\nu=$ resonance frequency of I). The true shielding tensor $\sigma(I)$ is then evaluated by averaging over the subspectra. As a further advantage, MAS spectra are obtained under "high-resolution" conditions. This often allows the treatment of overlapping resonances which can occur if a sample contains several like nuclei in different chemical environments, or in the presence of impurities.

The severe limitation of solid-state NMR studies of randomly oriented samples, viz., the loss of the directional information, is frequently overcome by comparison of the experimental results with calculated shielding data. Past experience with ${ }^{13} \mathrm{C}$ shielding tensors indicated that the tensor orientation is subject to much less variation than the actual magnitudes of the principal components in related compounds. $11,20,21$ It has been shown in these cases, as well as for the diphosphene $1,{ }^{3}$ that the orientation of the shielding tensor can be frequently derived from calculations of model compounds with much more accuracy than the actual values of the shielding themselves. In addition, it is expected that a theoretical analysis of phosphorus shielding in iminophosphines will be helpful to provide more detailed insight in certain underlying trends (e.g. substituent effects).

\section{Experimental Section}

Iminophosphines 5-16 were prepared as described elsewhere ${ }^{22-26}$ All NMR measurements were carried out on a Varian Unity-400 instrument operating at a field of $9.4 \mathrm{~T}$ and equipped with a high-speed MAS probehead. The samples were ground into a powder and transferred to 4-mm silicon nitride rotors, which were then closed with Kel-F caps. All manipulations were carried out in a glovebag under an $\mathrm{Ar}$ atmosphere. The samples were found to be stable in the closed rotors over a period of several days, without any necessity for further precautions.

MAS spectra were recorded at spinning rates between 7 and $10 \mathrm{kHz}$ at a ${ }^{31} \mathrm{P}$ resonance frequency of $161.9 \mathrm{MHz}$. No cross polarization was employed in order to prevent inhomogeneous excitation over the range of the shielding tensor. ${ }^{27}$ All spectra were acquired under high-power ${ }^{1} \mathrm{H}$ decoupling and ${ }^{31} \mathrm{P} \pi / 2$ pulse widths of $3-4 \mu \mathrm{s}$, with relaxation delays of $60-120 \mathrm{~s}$. Typical measurements required between 100 and 700 transients. Chemical shifts are referenced to external $85 \% \mathrm{H}_{3} \mathrm{PO}_{4}$ and are given in the $\delta$-scale $\left(\delta\left(\mathrm{H}_{3} \mathrm{PO}_{4}\right)=0\right)$. Base-line correction and deconvolution of the sidebands were performed for all spectra using both the spectrometer software and the built-in features of the MASNMR program package; ${ }^{28}$ both methods gave identical results. Isotropic chemical shifts were determined via moment analysis in MASNMR; in ambiguous cases, the values were checked by comparison of MAS spectra taken at different spinning speeds. The principal components of the shielding tensor were evaluated by analysis of the spinning sideband

(19) Gobetto, R.; Harris, R. K.; Apperley, D. C. J. Magn. Reson. 1992, 96, 119 .

(20) Facelli, J. C.; Orendt, A. M.; Beeler, A. J.; Solum, M. S.; Depke, G.; Malsch, K. G.; Downing, J. W.; Farvathi, S. M.; Grant, D. M.; Michl, J. J. Am. Chem. Soc. 1985, 107, 6479 .

(21) Facelli, J. C.; Orendt, A. M.; Solum, M. S.; Depke, G.; Grant, D. M.; Michl, J. J. Am. Chem. Soc. 1986, 108, 4268.

(22) (a) Niecke, E.; Lysek, M.; Symalla, E. Chimia 1986, 40, 202. (b) Romanenko, V. D.; Drapailo, A. B.; Ruban, A. V.; Markovskii, L. N. Z $h$. Romanenko, V. D.; Drapailo, A.
Obshch. Khim. 1986, $56,2792$.

(23) Hitchcock, P. B.; Lappert, M. F.; Rai, A. K.; Williams, H. D. J. Chem. Soc., Chem. Commun. 1986, 1633.

(24) (a) Niecke, E.; Detsch, R.; Nieger, M. Chem. Ber. 1990, 123, 797. (b) Detsch, R. Ph.D. Thesis, Universitāt Bonn, 1992.

(25) (a) Gãrrtner-Winkhaus, C. Ph.D. Thesis, Universităt Bonn, 1992. (b) Niecke, E.; Nieger, M.; Gārtner-Winkhaus, C.; Kramer, B. Chem. Ber. 1990, 123,477 .

(26) (a) Niecke, E.; Nieger, M.; Reichert, F. Angew. Chem., Int. Ed. Engl. 1988, 27, 1715. (b) Reichert, F. Ph.D. Thesis, Universitāt Bonn, 1990.

(27) Jeschke, G. Diploma Thesis, Technische Universitāt Dresden, 1992.

(28) Jeschke, G. MASNMR; Technische Universität Dresden, 1992. 
intensities using MASNMR ${ }^{28}$ as well as a similar computer program developed in-house; ${ }^{29}$ both programs are based on iterative adaptations of the method of Herzfeld and Berger. ${ }^{16}$ The principal components were indicated according to their magnitude, with $\delta_{11} \geq \delta_{22} \geq \delta_{33}$; given shifts are typically accurate to within $\pm 0.5 \mathrm{ppm}$ for $\delta_{\text {iso }}$ and $\pm 25 \mathrm{ppm}$ for $\delta_{t i t}$.

IGLO' calculations of the nuclear magnetic shielding tensors for the parent compounds 17-21 were performed with both "experimental" $6,22-26$ and ab-initio calculated (energy optimization using a DZP (double- $\xi+$ polarization) basis set at the SCF level ${ }^{30}$ ) geometries for 17-20; for 21, only the "experimental" geometry 24 was available. "Experimental" geometries were based on the X-ray structure data for the heteroatom skeleton; peripheral hydrogen atoms were attached at the observed bond angles, but with standard bond distances as derived from the SCFoptimized structures $\left(r(\mathrm{PH}) 1.43 \AA ; r(\mathrm{NH}) 1.01 \AA ; r(\mathrm{CH}) 1.10 \AA^{30}\right)$. The basis set used for the IGLO calculations was of TZP quality (basis II as described in ref 9c). The calculated (absolute) shieldings were converted to chemical shifts using the reference data by Jameson et al. ${ }^{31}$

\section{Results}

MAS Line Shapes. With the exception of $\mathbf{8}$, the sideband manifolds in the ${ }^{31} \mathrm{P}-\mathrm{MAS}$ spectra of 6-16 display symmetric Gaussian or mixed Lorentzian/Gaussian line shapes with halfwidths of $200-600 \mathrm{~Hz}$; significantly sharper lines $(70 \mathrm{~Hz})$ are only observed for 5 . No asymmetric splittings or shoulders are detected, indicating that the magnitude of residual quadrupolar splittings is smaller than the line widths. This is corroborated by comparison of the spectra of the isotopomers $14 \mathrm{a}, \mathrm{b}$, showing that replacement of ${ }^{14} \mathrm{~N}(I=1)$ by ${ }^{15} \mathrm{~N}(I=1 / 2)$ has no visible effect on the line shape. The MAS spectrum of $\mathbf{8}$ is unique, as the resonance lines appear as strongly overlapping asymmetric doublets (relative intensities approximately $2: 1$ for the center band) with a splitting of $440 \mathrm{~Hz}$.

Chemical Shift Tensors. The failure to resolve any residual dipolar splittings prevents the identification of the subspectra associated with different spin states of the quadrupolar nuclei and thus precludes spectral analysis in terms of effective shielding tensors. On the other hand, since the contribution from each subspectrum to a particular spinning sideband will vary somewhat because of the change in $\sigma^{\prime}$, a tensor analysis based on the integral sideband intensities may be susceptible to systematic errors. However, as the variation in $\sigma_{1}^{\prime}$ depends on the effective dipolar coupling constant $D^{\prime}, 19$ the relative deviations will become small when the overall shielding anisotropy significantly exceeds $D^{\prime}$ and at higher magnetic fields. For the cation $[\mathrm{P} \equiv \mathrm{N}-\text { aryl }]^{+}, 4$ $\left(D^{\prime}=960 \mathrm{~Hz}^{8}\right)$, the maximum difference in effective shielding anisotropies between the \pm 1 spin states of ${ }^{14} \mathrm{~N}$ is estimated according to Gobetto et al. ${ }^{19}$ as $12 \mathrm{ppm}$ at a field of $9.4 \mathrm{~T}$. Even if the approximate nature of this value must be conceded, owing to the nonaxiality of the shielding tensor, ${ }^{8}$ differences of this magnitude are small, as compared to the shielding anisotropy of $581 \mathrm{ppm}^{8}{ }^{8}$ Regarding that the shift anisotropies in iminophosphines are of similar size, the errors introduced by complete neglect of the residual dipolar effects can be tolerated. Heuristic justification of this approach is given by the spectral data of $14 \mathbf{a}, \mathbf{b}$. Here, shielding tensor analysis based on integral sideband intensities yields identical results within experimental error for both isotopomers, thus demonstrating that the effects of residual dipolar coupling with ${ }^{14} \mathrm{~N}$ are essentially negligible.

The principal components of the shielding tensor $\left(\delta_{l i}\right)$ as well as isotropic chemical shifts $\left(\delta_{\text {iso }}\right)$ of 5-16, as obtained by analysis of the sideband intensities according to the method of Herzfeld and Berger, ${ }^{16}$ are summarized in Tables 1 and 2, respectively. With the exception of 7 and 9 , where the two inequivalent ${ }^{31} \mathrm{P}$ nuclei give rise to separate resonances, all spectra display a single set of sidebands, which is consistent with the crystallographic equivalency of all molecules in the unit cell. Scalar couplings $\left({ }^{1} J_{\mathrm{PN}},{ }^{2} J_{\mathrm{PP}}\right)$ are not resolved, so that no corrections for $J$-anisotropy

(29) Gudat, D. Unpublished results.

(30) Schoellet, W. W.; Busch, T.; Niecke, E. Chem. Ber. 1990, 123, 1653.

(31) Jameson, C. J.; De Dios, A.; Jameson, A. K. Chem. Phys. Lett. 1990. $167,574$.
Table 1. ${ }^{31} \mathrm{P}$ Chemical Shift Tensor Data for $\mathrm{R}-\mathrm{P}=\mathrm{N}-$ aryl (aryl $\left.=\mathrm{C}_{6} \mathrm{H}_{2} \mathrm{tBu} \mathrm{B}_{3}\right), 5-16^{a}$

\begin{tabular}{|c|c|c|c|c|c|c|}
\hline & $\mathbf{R}$ & $\begin{array}{l}\text { double-bond } \\
\text { conformation }\end{array}$ & $\delta_{11}$ & $\delta_{22}$ & $\delta_{33}$ & $\delta_{i s o}$ \\
\hline 5 & $\mathrm{tBu}$ & $E$ & 1035 & 297 & 130 & 487 \\
\hline 6 & $\mathrm{Ph}_{3} \mathrm{Sn}(\mathrm{tBu}) \mathrm{N}$ & $E$ & 719 & 225 & 94 & 346 \\
\hline \multirow[t]{2}{*}{7} & aryl $-\mathrm{N}=\mathrm{P}(\mathrm{tBu}) \mathrm{N}$ & $E$ & 742 & 147 & 84 & 324 \\
\hline & & $E$ & 685 & 136 & 133 & 318 \\
\hline 8 & aryl-NH & $E$ & 628 & 124 & 90 & 281 \\
\hline \multirow[t]{2}{*}{9} & aryl $-\mathrm{N}=\mathrm{P}(\mathrm{Ad}) \mathrm{N}$ & $E$ & 758 & 171 & 76 & 335 \\
\hline & & $z$ & 441 & 84 & 48 & 191 \\
\hline 10 & $\mathrm{Ph}_{2} \mathrm{~N}$ & $\bar{Z}$ & 354 & 87 & -9 & 144 \\
\hline 11 & $\begin{array}{l}\mathrm{C}_{12} \mathrm{H}_{8} \mathrm{~N} \\
\text { (carbazolyl) }\end{array}$ & $Z$ & 385 & 75 & -36 & 141 \\
\hline 12 & $\mathrm{tBu}_{2} \mathrm{C}=\mathrm{N}$ & $E$ & 478 & 122 & 17 & 206 \\
\hline 13 & $\begin{array}{l}\mathrm{C}_{12} \mathrm{H}_{8} \mathrm{C}=\mathrm{N} \\
\text { (fluorenylimino) }\end{array}$ & $Z$ & 254 & 128 & 8 & 130 \\
\hline $14 \mathrm{a}$ & $\mathrm{Cl}$ & $Z$ & 445 & 129 & -140 & 145 \\
\hline $14 \mathrm{~b}$ & $\mathrm{Cl}\left({ }^{15} \mathrm{~N}\right)$ & $\bar{Z}$ & 446 & 131 & -143 & 145 \\
\hline 15 & $\mathrm{Br}$ & $\boldsymbol{Z}$ & 400 & 125 & -104 & 140 \\
\hline 16 & I & & 276 & 192 & -167 & 100 \\
\hline
\end{tabular}

${ }^{a}$ Reference: $\delta\left(\mathrm{H}_{3} \mathrm{PO}_{4}\right)=0.0$; negative values indicate increased shielding.

Table 2. Isotropic ${ }^{31} \mathrm{P}$ Chemical Shifts of $\mathrm{R}-\mathrm{P}=\mathrm{N}-$ aryl (aryl $=$ $\left.\mathrm{C}_{6} \mathrm{H}_{2} \mathrm{tBu}_{3}\right), 5-16$, in the Solid State and in Hydrocarbon Solution ${ }^{a}$

\begin{tabular}{|c|c|c|c|c|c|}
\hline & $\mathbf{R}$ & $\begin{array}{l}\text { double-bond } \\
\text { conformation }\end{array}$ & $\delta_{\text {soln }}$ & $\delta_{\text {iso }}$ & $\Delta \delta$ \\
\hline 5 & $\mathrm{tBu}$ & $E$ & 482 & 487 & -5 \\
\hline 6 & $\mathrm{Ph}_{3} \mathrm{Sn}(\mathrm{tBu}) \mathrm{N}$ & $E$ & 328 & 346 & -18 \\
\hline \multirow[t]{2}{*}{7} & aryl $-\mathrm{N}=\mathrm{P}(\mathrm{tBu}) \mathrm{N}$ & E & 272 & 324 & -52 \\
\hline & & E & & 318 & -46 \\
\hline 8 & aryl-NH & $E$ & 272 & 281 & -9 \\
\hline \multirow[t]{2}{*}{9} & aryl $-\mathrm{N}=\mathrm{P}(\mathrm{Ad}) \mathrm{N}$ & $E$ & 306 & 335 & -29 \\
\hline & & $\bar{Z}$ & 233 & 191 & 42 \\
\hline 10 & $\mathrm{Ph}_{2} \mathrm{~N}$ & $z$ & 198 & 144 & 54 \\
\hline 11 & $\begin{array}{l}\mathrm{C}_{12} \mathrm{H}_{8} \mathrm{~N} \\
\text { (carbazolyl) }\end{array}$ & $\boldsymbol{Z}$ & 186 & 141 & 45 \\
\hline 12 & $\mathrm{tBu}_{2} \mathrm{C}=\mathrm{N}$ & $E$ & 178 & 206 & -28 \\
\hline 13 & $\begin{array}{l}\mathrm{C}_{12} \mathrm{H}_{8} \mathrm{C}=\mathrm{N} \\
\text { (fluorenylimino) }\end{array}$ & $z$ & 124 & 130 & -6 \\
\hline 14a & $\mathrm{Cl}$ & $z$ & 136 & 145 & -9 \\
\hline 15 & $\mathrm{Br}$ & $\bar{z}$ & 162 & 140 & 22 \\
\hline 16 & I & & 218 & 100 & 118 \\
\hline
\end{tabular}

- Reference: $\delta\left(\mathrm{H}_{3} \mathrm{PO}_{4}\right)=0.0$; negative values indicate increased shielding. ${ }^{b}$ Double-bond configuration in the solid state.

need to be made; furthermore, no irregular intensity distributions associated with strong coupling effects ${ }^{14}$ are visible. The isotropic shifts in the solid state $\left(\delta_{\text {iso }}\right)$ differ in several cases considerably from the solution values $\left(\delta_{\text {roin }}\right)$ (Table 2$)$, the most pronounced deviation $(118 \mathrm{ppm})$ being observed for 16 . While all tensors have the typical large anisotropies of multiple bond systems in common, characteristic trends in chemical shielding following changes in both substitution and double-bond geometry are evident. The magnitude of these effects clearly exceeds the observed variations between individual derivatives belonging to the same class (e.g. different $R$ in $R_{2} N$ ).

Both the most deshielded value fo $\delta_{\text {iso }}$ and the largest chemical shift anisotropy is observed for 5 , which exhibits alkyl substitution at phosphorus and $(E)$-configuration of the double bond. The shielding tensor displays a marked negative axiality, ${ }^{16}$ which is a common feature for most ( $p-p) \pi$-double-bond systems $s^{3,5,11}$ and is also shared by the majority of the iminophosphines included in this study. Formal replacement of the alkyl by amino substituents (6-9) or ketimino substituents (12) leads to incresaed shielding of all three principal components, although to a different extent. By far the largest effect is found on $\delta_{11}$, which is upfield shifted by some 300 ppm in 6-9 as compared to 5 . $\delta_{22}$ experiences much less variation ( $\Delta \delta 100-200 \mathrm{ppm})$, while the changes in $\delta_{33}$ have to be considered insignificant $\left(\delta_{33}=130 \mathrm{ppm}\right.$ for $5,76-133$ ppm for 6-9). As a conseqeunce, replacement of the alkyl group by an amino group significantly reduces the overall shielding anisotropy as well as the difference between $\delta_{22}$ and $\delta_{33}$, even to 


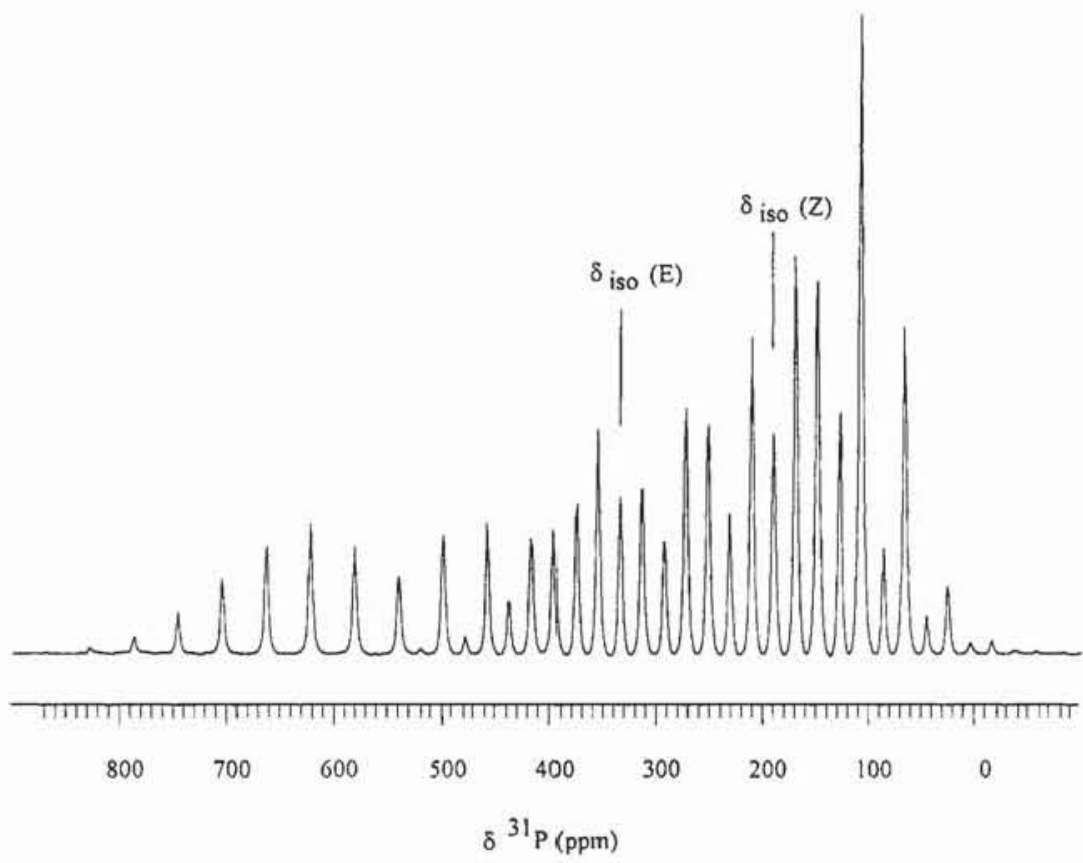

Figure 1. ${ }^{31} \mathrm{P}-\mathrm{MAS}$ spectrum $(161.9 \mathrm{MHz})$ of 9 at $6.59-\mathrm{kHz}$ rotation frequency. The spectrum clearly shows the two spinning sideband manifolds with characteristically different overall shielding anisotropies. The isotropic lines are marked and assigned to the two different sites.

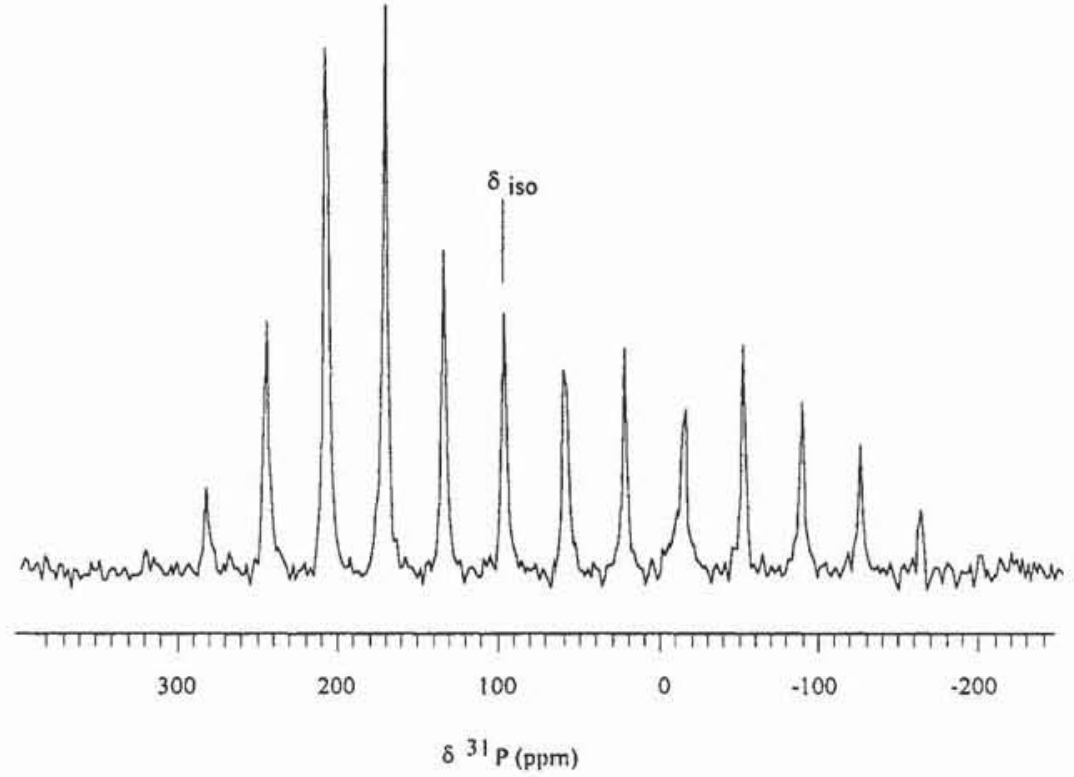

Figure 2. ${ }^{31} \mathrm{P}-\mathrm{MAS}$ spectrum (161.9 MHz) of 16 at $6.14-\mathrm{kHz}$ rotation frequency. The isotropic line is marked.

the extent that an essentially axial tensor is found for one of the phosphorus nuclei in 7.

In addition to the substituent effect, a further increase in shielding of $\delta_{11}$ by some $200-300 \mathrm{ppm}$ is associated with a change from $(E)-(6-9)$ to the $(Z)$-configuration of the double bond (9-11). Systematic, albeit much smaller, changes are evident for the other components. The effect is most impressively demonstrated in the case of 9 (Figure 1). Since both phosphorus nitrogen double bonds bear identical substituents, the substantial chemical shift difference by $317 \mathrm{ppm}$ for $\delta_{11}$ is in this case exclusively attributable to the different stereochemistries.

In ketimino-substituted derivatives, $\delta_{11}$ is found at considerably higher field, as in amino-iminophosphines ( $\Delta \delta 550 \mathrm{ppm}$ between 12 and 5), while the other components are only slightly more shielded. Consequently, the overall shielding anisotropy is further significantly reduced. Comparison of the data of 12 and 13 reveals that the configuration influence on the shielding tensor is still evident, but the magnitude of the chemical shift differences $(\Delta \delta$ $224 \mathrm{ppm}$ for $\delta_{11}$ ) is smaller than for amino-iminophosphines.

Shielding tensors very similar to each other are found for the P-halogen-substituted derivatives, 14 and 15. The values of $\delta_{11}$ and $\delta_{22}$ are comparable to $(Z)$-amino-iminophosphines; however, $\delta_{33}$ is considerably more shielded. As a consequence, the chemical shift anisotropy is increased and the observed tensor axialities are close to 0 . Still substantially higher shielding of $\delta_{33}$ is found for the P-iodo compound, 16. Since at the same time $\delta_{11}$ is shifted to higher and $\delta_{22}$ to lower field, the tensor adopts a unique positive axiality (Figure 2). On the whole, its shape is essentially the same as for the cation $4^{8}$ (see below), with the single difference of a somewhat reduced shielding anisotropy.

Model Calculations. The calculated tensor data are summarized in Table 3. The SCF-optimized conformations of 1720 exhibit planar symmetry, requiring that two of the principal components of the shielding tensor are confined within this plane 
Table 3. Principal Components and Orientation of Calculated Shielding Tensors for Iminophosphines $\mathrm{R}-\mathrm{Pm}=\mathrm{NH}, \mathbf{1 7}^{-21^{a}}$

\begin{tabular}{|c|c|c|c|c|c|c|c|c|c|}
\hline & $\mathbf{R}$ & $\begin{array}{l}\text { double-bond } \\
\text { conformation }\end{array}$ & geometry ${ }^{b}$ & $\delta_{11}$ & $\delta_{22}$ & $\delta_{33}$ & $\delta_{\text {ino }}$ & $\begin{array}{l}\text { out-of-plane } \\
\text { component }\end{array}$ & angle $\alpha$ (deg) \\
\hline \multirow[t]{2}{*}{17} & $\mathrm{H}$ & $E$ & scf & 1546.1 & 188.7 & 40.4 & 591.6 & $\delta_{33}$ & 120.2 \\
\hline & & $\boldsymbol{z}$ & scf & 899.7 & 327.1 & 12.1 & 409.6 & $\delta_{33}$ & 121.4 \\
\hline \multirow[t]{3}{*}{18} & $\mathrm{H}_{3} \mathrm{C}$ & $E$ & scf & 1429.6 & 309.3 & 33.8 & 590.9 & $\delta_{33}$ & 124.0 \\
\hline & & & expd & 1583.3 & 249.1 & 59.6 & 630.7 & $\delta_{33}$ & 129.9 \\
\hline & & $z$ & scf & 863.9 & 400.1 & 11.6 & 425.2 & $\delta_{33}$ & 127.7 \\
\hline \multirow[t]{4}{*}{19} & $\mathrm{H}_{2} \mathrm{~N}$ & $\bar{E}$ & scf & 963.8 & 103.4 & 72.6 & 379.8 & $\delta_{33}$ & 111.2 \\
\hline & & & $\exp ^{e}$ & 1013.8 & 94.8 & 51.4 & 386.7 & $\delta_{33}$ & 105.8 \\
\hline & & $Z$ & sef & 604.0 & 78.7 & 47.4 & 243.4 & $\delta_{33}$ & 118.3 \\
\hline & & & exp' & 394.0 & 42.1 & 15.4 & 150.5 & $\delta_{22}$ & 123.6 \\
\hline \multirow[t]{2}{*}{20} & $\mathrm{Cl}$ & $z$ & sef & 700.0 & 153.8 & -110.7 & 247.7 & $\delta_{22}$ & 114.2 \\
\hline & & & exps & 441.8 & 108.8 & -231.2 & 106.5 & $\delta_{22}$ & 115.8 \\
\hline \multirow[t]{2}{*}{21} & $\mathrm{HN}=\mathrm{P}-\mathrm{NH}$ & $E$ & $\exp ^{h}$ & 1051.7 & 88.8 & 40.3 & 393.6 & $\delta_{22}$ & 112.4 \\
\hline & & $\bar{Z}$ & & 466.5 & 48.5 & 12.9 & 176.0 & $\delta_{22}$ & 126.3 \\
\hline
\end{tabular}

- The calculated absolute shieldings $\sigma_{U}$ were converted to chemical shifts $\delta_{\| l}$ according to Jameson, de Dios, and Jameson. Reference: $\sigma\left(\mathrm{H}_{3} \mathrm{PO}_{4}\right)$ $=328.35 .{ }^{31} \mathrm{~b}$ scf $=$ scf-optimized geometry $(\mathrm{ref} 30)$; exp = experimental core geometry with attached hydrogen atoms; see text. ${ }^{c}$ In plane angle between $\delta_{11}$ and the PN double-bond axis:

$$
\overbrace{R}^{P} \sigma^{N H}
$$

d Based on X-ray structure of $\mathrm{Et}_{3} \mathrm{CP}=\mathrm{N}$-aryl. ${ }^{38}$ e Based on X-ray structure of $7 .{ }^{24} f$ Based on X-ray structure of $10 .{ }^{25} \varepsilon$ Based on X-ray structure of $14 \mathrm{a}^{26} h$ Based on X-ray structure of 9.24

("in-plane"), while the third one is perpendicular ("out-of-plane"). In all cases but 20 , this component is the most shielded one $\left(\delta_{33}\right)$. For 20, the out-of-plane component is $\delta_{22}$, leaving $\delta_{33}$ as the inplane component closest to the direction of the phosphorusnitrogen bond. The most deshielded component $\left(\delta_{11}\right)$ is always in-plane and points close to the direction of the $\mathrm{P}-\mathrm{X}$ single bond. The angle $\alpha$ spanned by $\delta_{11}$ and the double-bond axis is generally somewhat larger than the phosphorus valence angle. The size of $\alpha$ changes by less than $\pm 10^{\circ}$ for all calculated structures, indicating that the orientation of the principal axis system changes indeed much less than the magnitude of the shielding in each direction. For the heteronuclear substituted derivatives 18-20, the variations in $\alpha$ appear to be loosely correlated to the change in the XPN angle.

Even if in the "experimental" structures the planar symmetry is broken, the measured dihedral (XPNH) angles differ from the

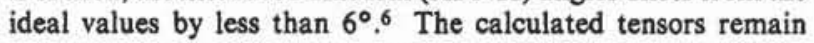
closely aligned with respect to the corresponding best-fit planes, and in principle the same orientations of the principal axes are derived as for the case of SCF-optimized geometry. An exception is constituted by $(\mathrm{Z})-\mathrm{H}_{2} \mathrm{~N}-\mathrm{P}=\mathrm{NH}, 19$, where the assignment of $\delta_{22}$ (out-of-plane) and $\delta_{33}$ (in-plane) is reversed as compared to the SCF-optimized structure. The same tensor orientation, which equals that of the $\mathrm{Cl}$ derivative 20, is also found for both phosphorus shielding tensors in $\mathbf{2 1}$.

For a further discussion of trends in chemical shift data it is necessary to establish a correspondence between the measured and calculated shieldings for the individual compounds. The unique downfield shift of $\delta_{11}$ in 5-16 allows its unequivocal assignment as the in-plane tensor component lying closest to the $\mathrm{P}-\mathrm{X}$ single bond in all cases. For alkyl-substituted (5) or halogen-substituted derivatives (14-16), the discrimination between the remaining components in the calculations is considered as satisfactory to establish $\delta_{33}(5)$ and $\delta_{22}(14-16)$, respectively, as the out-of-plane component. The calculated values of $\delta_{22}$ and $\delta_{33}$ for amino-iminophosphines are much closer, and their difference is of similar magnitude as the deviation between experimental $(8,10)$ and calculated (19) data, making an assignment exceedingly difficult. As further calculations on $(E)$ $\mathrm{H}_{2} \mathrm{~N}-\mathrm{P}=\mathrm{N}-\mathrm{H}(19)$ revealed that moderate alterations of bond distances or angles leave the tensor orientation unchanged, it is suggested that the alignment of the principal axes in the $(E)$ isomers $6-8$ and 12 is presumably similar to alkyl derivatives, with $\delta_{33}$ being out-of-plane. In contrast, no unambiguous assignments can be made for 9 and the $(Z)$-isomers 10,11 , and 13 , where, according to the calculations, the out-of-plane principal axis may be either $\delta_{22}$ or $\delta_{33}$, depending on the individual geometry.
Naturally, the numerical coincidence between experimental and calculated values of principal components is not very good as is expected given the considerable simplifications of the model compounds and the known tendency of the Hartree-Fock-based methods (such as IGLO) to overestimate strong deshielding contributions. Nonetheless, the effect of structural variations on the shielding is clearly evident. The most significant variations of shielding parameters are induced by changes of substitution or double-bond configuration. Using the indication for the experimentally determined tensor components as described above, the observed trends can be reproduced by the calculated data. The influence of the double-bond stereochemistry on the shielding tensor is not confined to amino-substituted derivatives, but seems to be a general feature of phosphorus-nitrogen double-bond systems. Variation of bond distances or angles ("experimental" vs SCF-calculated geometry) results in less pronounced, but yet significant, changes of calculated shieldings, thus giving emphasis to the sensitivity of the shielding tensor of a nucleus as a probe for changes in the three-dimensional electronic structure of its surroundings.

\section{Discussion}

Quadrupolar Splittings. Recently, Wasylishen and co-workers ${ }^{32}$ reported quadrupolar splittings of $140 \pm 20 \mathrm{~Hz}$ in the ${ }^{31} \mathrm{P}-\mathrm{MAS}$ spectra of monophosphazenes $\mathrm{Ph}_{3} \mathrm{P}=\mathrm{NR}^{\prime}\left(\mathrm{R}^{\prime}=\mathrm{Ph}\right.$, tolyl) at a magnetic field strength of $4.7 \mathrm{~T}$, which can be extrapolated ${ }^{18}$ to values around $70 \mathrm{~Hz}$ at the higher field $(9.4 \mathrm{~T})$ applied here. Whereas the detection of quadrupolar interactions of this magnitude in the MAS spectra of 6-16 is precluded by the large line widths, the residual quadrupolar splitting must be much smaller in the case of 5 . Assuming similar phosphorus-nitrogen distances and comparable electric field gradients at nitrogen for both phosphazenes and iminophosphines, this effect may be attributed to a lower nuclear quadrupole coupling constant $(\chi)$ or a different geometry relation of the coupling tensors, or a combination of both, in the iminophosphine $\mathbf{5}$. In the case of $\mathbf{8}$, neither the crystal structure ${ }^{26 a}$ nor solid-state ${ }^{13} \mathrm{C}-\mathrm{NMR}$ spectra ${ }^{266}$ give evidence for magnetic nonequivalent molecules in the asymmetric unit, so that a quadrupolar origin of the observed line splitting seems likely. However, a simple interpretation in terms of residual dipolar coupling is questionable, since the magnitude of the splitting would require a substantially increased value of $\chi$ in this case, for which no immediate reason can be seen. Regarding that a deeper analysis of the quadrupolar

(32) Power, W. P.; Wasylishen, R. E.; Curtis, R. D. Can. J. Chem. 1989, $67,1989$. 
interaction, even in terms of a simple perturbation treatment, ${ }^{18}$ would require knowledge of several geometric as well as energetic parameters which is presently not available, no further interpretation of the effect is attempted.

Trends in Chemical Shift Tensors. It is generally acknowledged that in the case of multiple bond systems the variations in the shielding tensor components are dominated by changes in the paramagnetic term, $\sigma_{\mathrm{p}}{ }^{33}$ The magnitude of $\sigma_{\mathrm{p}}$ strongly depends on the availability of excited states which are connected with the ground state by magnetic-dipole-allowed transitions; large deshieldings are generally associated with low transition energies. For iminophosphines, the lowest magnetically allowed transition corresponds to the $n(P)-\pi^{*}(P N)$ electronic excitation, whose energy has been shown by previous spectroscopic studies to be sensitively affected by the electronic structure of the $\pi$-bond. 2,6,34 Due to the nature of the angular momentum operators in the expression for $\sigma_{\mathrm{p}}$, any changes in the shielding tensor related to variation of the $n-\pi^{*}$ excitation energy should be strongest in a direction which is perpendicular to both of the two axes through the lobes of the phosphorus lone pair and the $\pi^{*}$-orbital; this approximately coincides with the direction of the $\mathrm{P}-\mathrm{X}$ single bond. The least effect is expected on the out-of-plane principal component which is parallel to the lobe of the $\pi^{*}$-orbital; within the molecular plane, the least changes of $\sigma_{\mathrm{p}}$ should occur parallel to the lobe of the phosphorus lone pair.

On the basis of the suggested orientation of the shielding tensor in iminophosphines, the large increase in $\delta_{11}$ upon going from P-alkyl-iminophosphines (5) to amino-iminophosphines (6-11) is considered predominantly as a direct consequence of the corresponding increase in $n-\pi^{*}$ excitation energy, which results from both the perturbation of the double-bond system by $\pi$-conjugation ${ }^{34}$ and the higher ligand electronegativity. 2,30 This interpretation is further corroborated by analysis of individual orbital contributions to the shielding, which shows that the largest variations of $\delta_{11}$ arise indeed from the phosphorus nonbonding orbital. In this sense, the further increased shielding of $\delta_{11}$ in ketimine-substituted derivatives suggests a higher $\pi$-donating capability of ketimino groups as compared to amino groups. The same conclusion has been derived based on the crystal structure data of 12 and 13. ${ }^{25}$ Halogen substituents lead in the case of $\mathrm{Cl}$ and $\mathrm{Br}$ to slight deshielding of $\delta_{11}$, as compared to aminoiminophosphines of like stereochemistry, which is in accord with their lower $\pi$-donating capability.

In principle the same trends as for $\delta_{11}$ are observed for the second in-plane component in $\mathbf{5}$ and amino-iminophosphanes (611). However, since the angle between the tensor axis and the lobe of the phosphorus nonbonding orbital is now much smaller than $90^{\circ}$, transmission of changes in the electronic structure of the double bond on the shielding is less pronounced and the degree of variation is smaller than for $\delta_{11}$. Consequently, further contributions to the paramagnetic term $\sigma_{\mathrm{p}}$, which are associated with energetically higher electronic transitions, grow in importance, thus accounting for a larger spread of the observed data. The out-of-plane component is essentially collinear with the lobes of the $\pi$-orbital, and all paramagnetic contributions associated with excitations into the $\pi^{*}$-orbital nearly vanish. This provides an explanation for the higher shielding of this component as well as for its insensitivity with respect to electronic effects related to the $\pi$-system. Instead, both calculations and experimental data indicate that the shielding of the out-of-plane component decreases with a higher electronegativity of the ligand $\mathbf{X}$. Since larger polarity of the $\mathrm{P}-\mathrm{X}$ bond lowers the energy of the antibonding $\sigma^{*}(\mathrm{PX})$-orbital, this effect can be related to an increased magnitude of the paramagnetic term owing to the lower energy

(33) (a) Mason, J. In Multinuclear NMR; Mason, J., Ed.; Plenum Press: New York, 1987, p 335 ff. (b) Chesnut, D. B. In Phosphorus-31 NMR Spectroscopy in Stereochemical Analysis; Verkade, J. G., Quin, L. D., Eds.; VCH Publishers: Deerfield Beach, FL, 1987; p $185 \mathrm{ff}$

(34) Niecke, E.; Gudat, D.; Schoeller, W. W.; Rademacher, P. J. Chem. Soc., Chem. Commun. 1985, 1050. for the $\mathrm{n}(\mathrm{P}) \rightarrow \sigma^{*}(\mathrm{PX})$ charge-transfer transition. For 14-16, the comparatively long phosphorus-halogen distances lead to further amplification of this effect. 6.26

The observed large upfield shifts of $\delta_{33}$ in the halogenated compounds 14-16 need some further consideration, since an explanation based exclusively on differences in $n-\pi^{*}$ excitation energies seems unsatisfactory. As compared to their congeners 5-13, the crystal structures reveal substantially larger angles at nitrogen and shortening of the phosphorus-nitrogen bond distances, while the phosphorus angles are similar. ${ }^{6}$ Model calculations on $\mathrm{Cl}-\mathrm{P}=\mathrm{NH}$ with systematic variations of the geometry indicate that a higher nitrogen valence angle produces a marked additional shielding contribution for both in-plane components, while the out-of-plane component is deshielded. This behavior can be interpreted in terms of increasing phosphorusnitrogen triple-bond character. For a genuine phosphorusnitrogen triple bond $\left([\mathrm{H}-\mathrm{N} \equiv \mathrm{P}]^{+}\right)$, calculations revealed an axial shielding tensor with a highly shielded parallel component along the phosphorus-nitrogen bond. ${ }^{35}$ Perturbation of this system by an additional chlorine ligand is expected to lead to reorientation of this principal axis away from the phosphorus-nitrogen bond, together with a shift to lower field. The degeneracy of the perpendicular components is removed, with the now in-plane component experiencing an increasing paramagnetic contribution from the concomitant lowering of the $n-\pi^{*}$ transition energy. Comparison of the shielding tensors for 14-16 suggests a substantially higher degree of triple-bond character in the P-iodo derivative as compared to its lighter homologues, which is completely in accord with the observed differences in the molecular structures. ${ }^{6}$ For $\mathbf{1 4}$ and $\mathbf{1 5}$, the contribution of the triple-bonded resonance structure can be held responsible for the unique reorientation of the tensor axes with respect to the other types of iminophosphines.

The influence of the $(E / Z)$-configuration of the double bond is clearly reproduced by the calculations. The shielding tensors calculated for the $(E / Z)$-isomers of $\mathrm{HP}=\mathrm{NH}$ and $\mathrm{H}_{3} \mathrm{CP}=\mathrm{NH}$ demonstrate that the observed effect is not confined to amino. iminophosphines but is presumably a general quality of the phosphorus-nitrogen double bond. As for the substituent effect, closer analysis reveals the paramagnetic contribution to the shielding as the origin of this effect. The differences are most pronounced for $\delta_{11}$, and the stronger deshielding in the $(E)$-isomers is related to two dominant orbital contributions which are attributable to the phosphorus lone pair and the phosphorusnitrogen bonding orbitals; it should be noted that a different relative order is observed for the contributions originating from the P-X single bond. On the whole, the model calculations establish that the observed effect is a genuine geometry effect; however, no easy picture of its origin emerges from the data, and the calculations do not help to relate the effect to simple orbital interactions.

Isotropic Chemical Shifts. The substitution and $(E / Z)$ stereochemistry effects should be reflected in similar trends for $\delta_{\text {iso, }}$ as have been discussed above. However, as averaging over different contributions to a single value of $\delta_{\text {iso }}$ necessarily also averages over the different and possibly counteracting trends in individual principal components, the changes are less characteristic, and diagnostic information is lost to some extent. Nonetheless, as the largest changes in $\delta_{11}$ and $\delta_{22}$ can be associated with a common origin, viz., variation of $n-\pi^{*}$ transition energies, isotropic shifts still comprise a sensitive probe for changes in the electronic structure of the double-bond system, and comparison of $\delta_{\text {iso }}$ gives generally a very similar picture as derived from the analysis of individual principal components. This assumption is also corroborated by the observed correlation between solution shifts and $n-\pi^{*}$ excitation energies. $2,6,36$ In a similar way, $\delta_{\text {ivo }}$

(35) IGLO calculations of the shielding tensor of $[\mathrm{HN} \equiv \mathrm{P}]$ gave values of $338(\delta \perp)$ and $-626(\delta \|)$, respectively: Fleischer, U. Unpublished results.

(36) David, G.; Busch, T.; Radewacher, P.; Schoeller, W. W.; vou der Gőua, V.; Niecke, E. J. Chem. Soc., Faraday Trans. II, in press. 
Table 4. Comparison of Chemical Shielding Data of 5 and 16 with Known Phosphorus-Containing Multiple Bond Systems

\begin{tabular}{|c|c|c|c|c|c|c|c|}
\hline & & $\begin{array}{l}\text { double-bond } \\
\text { conformation }\end{array}$ & $\delta_{11}$ & $\delta_{22}$ & $\delta_{33}$ & $\delta_{\text {ino }}$ & $\mathrm{ref}$ \\
\hline $\begin{array}{l}\mathbf{5} \\
\mathbf{1} \\
\mathbf{2}\end{array}$ & $\begin{array}{l}\mathrm{tBuP}-\mathrm{N}-\mathrm{aryl} \\
\text { aryl-P=P-aryl } \\
\text { aryl-P }=\mathrm{C}\left(\mathrm{SiMe}_{3}\right)_{2}\end{array}$ & $\begin{array}{l}E \\
E \\
E\end{array}$ & $\begin{array}{r}1035 \\
1236 \\
819\end{array}$ & $\begin{array}{l}297 \\
249 \\
249\end{array}$ & $\begin{array}{r}130 \\
-3 \\
-31\end{array}$ & $\begin{array}{l}487 \\
494 \\
394\end{array}$ & $\begin{array}{l}3 \\
5\end{array}$ \\
\hline $\begin{array}{r}16 \\
3 \\
4\end{array}$ & $\begin{array}{l}\mathrm{IP}=\mathrm{N}-\text { aryl } \\
\text { aryl-C }=\mathrm{P} \\
\text { aryl-N } \equiv \mathrm{P}^{+} \mathrm{AlCl}_{4}^{-}\end{array}$ & & $\begin{array}{l}276 \\
229 \\
308\end{array}$ & $\begin{array}{l}192 \\
140 \\
196\end{array}$ & $\begin{array}{l}-167 \\
-274 \\
-273\end{array}$ & $\begin{array}{r}118 \\
31 \\
77\end{array}$ & $\begin{array}{l}5 \\
8\end{array}$ \\
\hline
\end{tabular}

responds to the change between the $(E)$ - and $(Z)$-geometry of the double bond; however, as the difference between corresponding stereoisomers is reduced to some $100 \mathrm{ppm}$, the effect if far less significant.

Comparison of isotropic shifts in solid state and solution further provides a valuable test for comparability of the molecular structures in both phases. The significant deviations between $\delta_{\text {ino }}$ and $\delta_{\text {soin }}$ observed in several cases (see Table 2) indicate that measurable differences exist between solid-state and solution structures. The clearest evidence in this regard is obtained in the case of 16, where solution NMR investigations indicate the presence of separated individual molecules, while the crystal structure clearly reveals distinct intermolecular association in the solid state. ${ }^{6}$ The deshielding of $\delta_{\text {noin }}$ by $118 \mathrm{ppm}$ may thus be taken as an indication that the triple-bond character as derived from the solid-state NMR data is substantially promoted by the intermolecular interactions in the solid, whereas the solution structure is presumably more closely related to that of aminoiminophosphines.

For the remaining compounds, the origin of the differences in isotropic shifts is less evident, but it is strongly suggested that the effect reflects distinct distortions of the molecular geometry. Experimental support for this hypothesis is gained from crystal structure studies, where it was shown in several cases that different solid modifications of the same iminophosphine may exhibit considerable variations in nitrogen bond angles as well as phosphorus-nitrogen bond distances. ${ }^{6}$ A similar effect could occur during the transition between solid state and solution. In addition, theoretical investigations predict generally low-energy barriers for nitrogen inversion in iminophosphines, $2,6,7 a, 30$ thus suggesting that dynamic $(E / Z)$-isomerization of the double bond in solution may be of importance. Direct NMR evidence for this process is provided by the observed equilibration of the two different phosphorus environments in 9.37 In general, however, the differences between $\delta_{\text {ino }}$ and $\delta_{\text {soin }}$ are smaller as those expected for a change in double-bond stereochemistry, indicating that the same double-bond configuration prevails in solution and in the solid state. An exception may be the case of 7 , where the considerably higher solution shift can be interpreted in terms of inversion of one of the two double bonds, resulting in a conformation which is similar to 9.

Comparison with Other Phosphorus-Containing Multiple Bond Systems. The shape of the shielding tensor of $\mathbf{5}$ is closely similar to the aryl-substituted diphosphene, $1{ }^{3}$ and phosphaalkene, $2,{ }^{5}$ respectively (Table 4$). \delta_{33}$ and, to a somewhat lesser extent, $\delta_{22}$ are markedly deshielded in 5 , possibly reflecting the influence of the higher electronegativity of the second element in the double bond ( $N$ vs $P$ or $C$ ), while $\delta_{11}$ is found to lie at an intermediate value. As a consequence, the shielding anisotropy in 5 is somewhat reduced with respect to its higher homologue, 1, yet still larger, as in the phosphaalkene. The orientation of the shielding tensor in $\mathbf{5}$ is in principle the same as in the diphosphene, displaying the most shielded component lying perpendicular to the nodal plane of the $\pi$-bond and the most deshielded one directed approximately along the $\mathrm{P}-\mathrm{X}$ single bond. ${ }^{3}$ The increased angle between $\delta_{11}$

(37) Niecke, E.; Detsch, R.; Nieger, M.; Reichert, F.; Schoeller, W. W. Bull. Soc. Chim. Fr. 1993, 130, 25.

(38) Barion, D.; Gărtner-Winkhaus, C.; Link, M.; Nieger, M.; Niecke, E. Chem. Ber. 1993, 126, 2187. and the double-bond axis for $\mathbf{5}$ is in accord with the observed larger bond angle at phosphorus. As has already been noted, ${ }^{3,5}$ the orientation of the shielding tensor strongly resembles known ${ }^{13} \mathrm{C}$ tensors of alkenes, however, with a significant increase in anisotropy.

According to both structural ${ }^{6}$ and NMR data, the P-iodoiminophosphine 16 exhibits considerable triple-bond character. This suggests that the observed shielding tensor should be comparable to that of aryl $-\mathrm{N} \equiv \mathrm{P}^{+}(4)^{8}$ and aryl $-\mathrm{C} \equiv \mathrm{P}(3),{ }^{5}$ featuring genuine phosphorus-carbon or phosphorus-nitrogen triple bonds, which is indeed the case. The reduced shielding anisotropy in 16 as compared to the cation $4^{8}$ results mainly from the less pronounced shielding of $\delta_{33}$, indicating ${ }^{5}$ that perturbation of the phosphorus-nitrogen $\pi$-system by interaction with iodide (16) leads to a more pronounced decrease in bond order, as compared to $\mathrm{AlCl}_{4}^{-}$(4). The close correspondence between the magnitude of the principal components can be considered as a further strong argument that, similar to that in 4 , the most shielded principal component of 16 is in-plane and close to the phosphorusnitrogen bond axis.

\section{Conclusions}

${ }^{31}$ P nuclear magnetic shielding tensors of the iminophosphines 5-16 may be determined from high-resolution MAS NMR measurements. In combination with ab-initio chemical shift calculations, which allow by comparison the approximate determination of the tensor orientation in the molecular frame, this provides a detailed understanding of the correspondence between electronic structure of the $\pi$-bond and chemical shielding. Two major interactions are identified which mediate the influence of substituents at the double bond on the shielding tensor and whose magnitude clearly exceeds that of further contributions to the total shielding. (i) Attachment of $\pi$-donor type ligands at phosphorus strongly reduces the paramagnetic shielding contribution, which can be related to a higher energy of the magnetically allowed $n-\pi^{*}$ electronic transition; this effect is strongest for the most deshielded principal component which is oriented closest to the $\mathrm{P}-\mathrm{X}$ single bond. The shielding in this direction is further subtly dependent on the stereochemistry of the double bond, allowing a discrimination between $(E)$ - and $(Z)$-configuration solely on the basis of the NMR data. (ii) The presence of strongly electronegative halogen ligands, which has been shown to result in increased nitrogen valence angles, ${ }^{30}$ gives rise to a large additional shielding contribution for the principal component closest to the phosphorus-nitrogen bond. This effect can be explained as a consequence of partial triple-bond character of the phosphorus-nitrogen bond and may lead to an unprecedented change in the orientation of the shielding tensor. Whereas for the majority of known double-bond systems the most shielded principal component is perpendicular to the nodal plane of the $\pi$-orbital, it is close to the direction of the phosphorus-nitrogen multiple bond in halogenated iminophosphines; amino-iminophosphines are a borderline case where either alternative may prevail. In the case of the P-iodo derivative 16, intermolecular association in the solid state produces perturbation of the $\pi$-system to an extent that the shielding tensor shows the characteristic features of a genuine triple-bond system. Comparison of isotropic chemical shifts gives evidence that this special situation is not preserved in solution.

Acknowledgment. This work was financially supported by the Deutsche Forschungsgemeinschaft (SFB 334) and the Fonds der Chemischen Industrie. Allocation of computer time on the CYBER 205 at the Ruhr Universităt Bochum and the Siemens/ Fujitsu SNI600 at the computer center of the RWTH Aachen is gratefully acknowledged. Thanks are due to Prof. Dr. G. Grossmann, Technische Universitāt Dresden, who kindly provided the MASNMR program. We further thank Prof. G. Grossmann and Dr. A. Sebald, Universität Bayreuth, for many helpful discussions. 